\title{
First Alignment of the CMS Tracker and Implications for the First Collision Data
}

\author{
Andrei V. Gritsan* \\ Department of Physics and Astronomy, Johns Hopkins University, Baltimore, MD, USA \\ E-mail: gritsanejhu.edu
}

\begin{abstract}
We present the first results of the full CMS Silicon Tracker alignment based on several million reconstructed tracks from the cosmic data taken during the commissioning runs with the detector in its final position. Implication for CMS physics performance is discussed. The all-silicon design of the tracking system of the CMS experiment is expected to provide $1-2 \%$ resolution for $100 \mathrm{GeV}$ tracks and an efficient tagging of $b$-jets. To achieve optimal performance the position and orientation of each of the 15148 silicon strip and 1440 silicon pixel modules need to be determined with a precision of several micrometers. For the modules well illuminated by cosmic ray particles, the ultimate precision has been achieved with data from the silicon modules traversed in-situ by charged muons used in combination with survey measurements. The achieved resolution in all five track parameters is controlled with data-driven validation of the track parameter measurements near the interaction region, and tested against prediction with detailed detector simulation. Outlook for expected tracking and physics performance with the first collisions is given.
\end{abstract}

European Physical Society Europhysics Conference on High Energy Physics, EPS-HEP 2009, July 16 - 222009

Krakow, Poland

\footnotetext{
${ }^{*}$ Speaker.
} 
Precise determination of the position of 1440 silicon pixel and 15148 silicon strip detector modules is a challenging task and one of the critical aspects for achieving the design track parameter resolutions in the CMS experiment. The CMS collaboration conducted a month-long data-taking exercise known as the Cosmic Run At Four Tesla (CRAFT) during October-November 2008. Prior to CRAFT and during the final installation phase of the experiment, a series of commissioning exercises to record cosmic ray events took place with the solenoid turned off from May to September 2008. A smaller number of cosmic ray events with the tracker participation were recorded compared with CRAFT. The alignment of about $15 \%$ of the CMS silicon strip tracker was also performed prior to that with data taken during stand-alone commissioning in 2007 [1].

The silicon pixel detector is composed of two sub-detectors, the Barrel and the two Endcaps in the forward regions. The pixel modules provide two-dimensional measurements of the hit position in the module planes, which effectively translate into three-dimensional measurements in space. The silicon strip detector is composed of four sub-detectors: the Tracker Inner and Outer Barrels, the Tracker Inner Disks, and the Tracker Endcaps. The two inner layers of both strip barrel and in several rings of the strip endcaps are equipped with double-sided modules; all other positions have single-sided modules.

Optical surveys taken during module construction and integration provide initial alignment parameters for many of the modules. Additionally, the survey information was used as constraints in the alignment procedure, described below. Coordinate Measuring Machine (CMM) data and photogrammetry have been used for the optical survey of the tracker components. While the former were used for measurements of the active elements, the latter were used for the alignment of larger structures.

An independent test of the silicon tracker alignment was provided by the Laser Alignment System (LAS), which uses a system of 40 infrared laser beams $(\lambda=1075 \mathrm{~nm})$ to survey the position of the large-scale structure elements of the tracker. The LAS measurements are available for 434 silicon strip modules, which are distributed over eight azimuthal sectors. The beam light is detected directly on the active area of the silicon sensor.

The alignment is an optimization problem that can be formulated in the context of linear least squares. Module position corrections ("alignment parameters") $\mathbf{p}$ are determined by minimizing a function

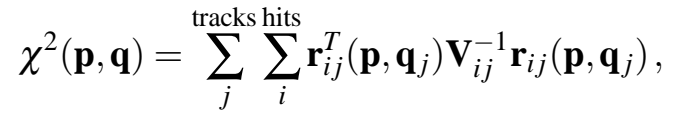

which can be expressed as the sum over all hits $i$ on all tracks $j$ and track parameters $\mathbf{q}_{j}$, assuming negligible correlations between hits. Track residuals $\mathbf{r}_{i j}$ are defined as the difference between the measured hit position and the trajectory impact point, and $\mathbf{V}_{i j}$ is their covariance matrix.

Two statistical methods were employed to solve the alignment problem. Both of them were previously applied to the CMS silicon strip tracker alignment during stand-alone commissioning [1]. The global alignment algorithm ("Millepede II") [2] minimizes the $\chi^{2}$ function in Eq. (1) by taking into account track and alignment parameters simultaneously. The local iterative algorithm ("Hits and Impact Points") [3] approximates Eq. (1) by assuming no track parameter q dependence. The track parameters and correlations between different modules are solved through iterations of the minimization procedure and refitting the tracks with new alignment constants after each iter- 

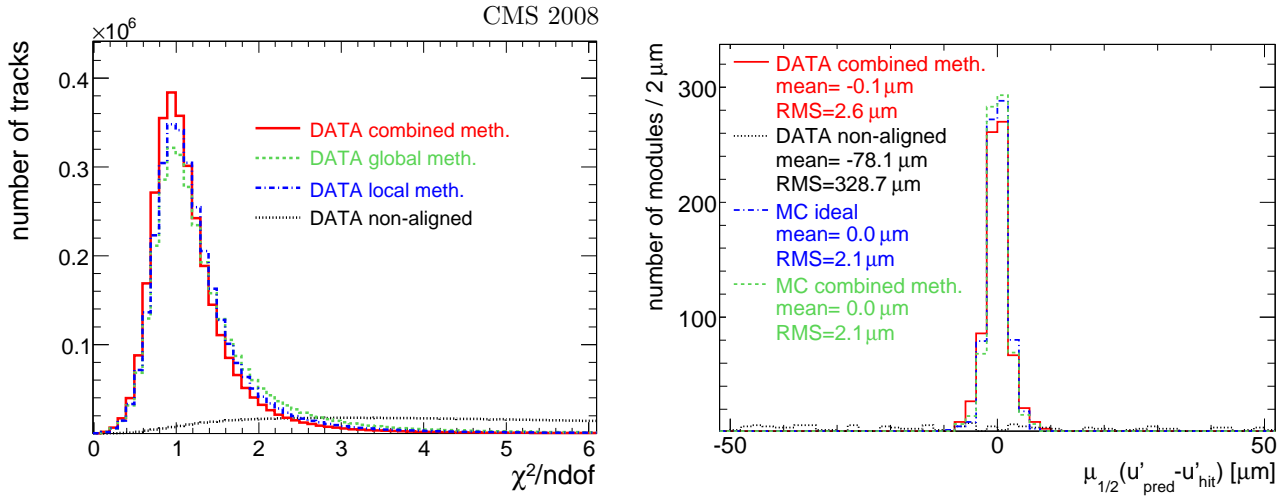

Figure 1: Distributions of the $\chi^{2}$ per degree of freedom of the tracks (left) for non-aligned, local method, global method, and combined method geometries; and distribution of the median of the residuals (right) for non-aligned, combined method in data, ideal and combined method in Monte Carlo simulation.
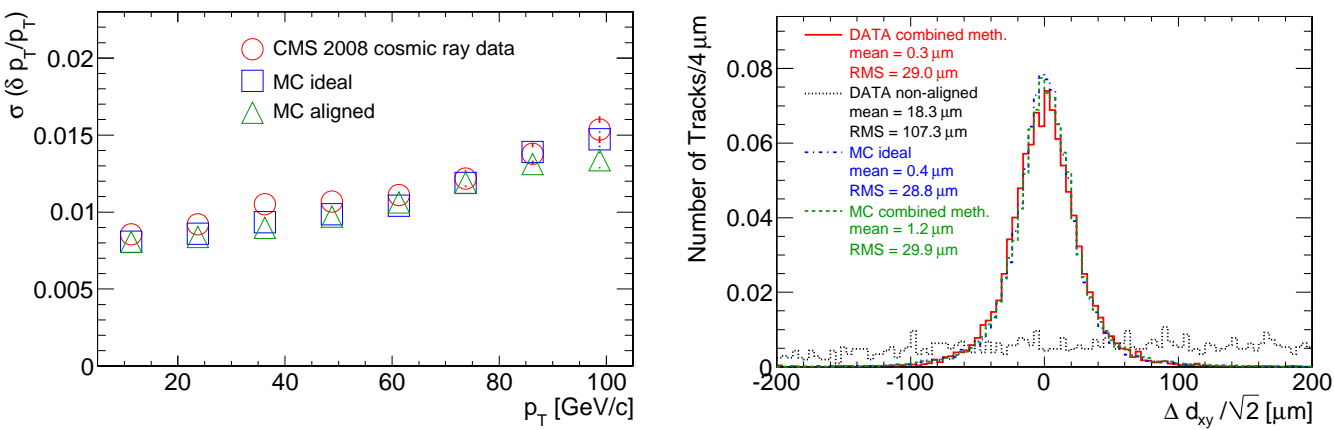

Figure 2: Resolution of the track parameters for the transverse momentum $\left(p_{T}\right)$ as a function of $p_{T}$ (left) and for the distance of closest approach in the transverse direction $d_{x y}$ (right). Two halves of cosmic tracks were used in this validation. The same geometries are shown as in the right plot of Fig. 1.

ation. The local iterative algorithm includes survey measurements in the formalism of Eq. (1), as described in Ref. [4].

After verifying that the two methods yielded consistent results, the final results were obtained by applying the two algorithms to CRAFT data in sequence in order to take advantage of their complementary strengths: first the global method allows the determination of alignment parameters, properly accounting for the correlations among them, in a single step, and then the local iterative method uses the full implementation of the Kalman filter track reconstruction algorithm adopted in CMS.

Several approaches were employed to validate the alignment results. The low-level quantities that were used in the $\chi^{2}$ minimization, such as residuals and the $\chi^{2} /$ ndof of the tracks, were monitored. The track $\chi^{2} /$ ndof distribution is shown in Fig. 1. The hit residual width is dominated by two effects other than alignment: track extrapolation uncertainties due to multiple scattering and hit position reconstruction uncertainties. Both of these effects are random, while misalignment leads to systematic shifts of the residuals. For this reason the distribution of the median $\mu_{1 / 2}$ of the residuals (DMR) is taken as the most appropriate measure of alignment and shown for the 
pixel detector in Fig. 1. Resolution of the track parameters is shown in Fig. 2. To check the statistical precision of track-based alignment a Monte Carlo simulation was also performed. Overall, there is significant improvement in the track reconstruction going from the geometry without any alignment, to the alignment using tracks with the local and the global method, and finally to the combined result. With respect to cosmic ray trajectories, the module positions were determined to a precision of 3-4 $\mu \mathrm{m}$ RMS in the barrel and 3-14 $\mu \mathrm{m}$ RMS in the endcaps in the most sensitive coordinate. However, systematic misalignment on large scale which affect $\chi^{2}$ only weakly remain a challenge and will be addressed with more data.

Experience gained in the alignment analysis of the silicon modules with cosmic ray particles is valuable in preparation for the full CMS tracker alignment with the data from LHC collisions, which is critical in achieving the physics goals of the CMS detector. Integration of measurements from cosmic and collision tracks, LAS, and survey will be critical for the optimal tracker alignment with the first data expected from LHC beam collisions. Optimization of the single-track resolution and reconstruction efficiency, $b$-tagging algorithm performance, resonance resolution, and as a result discovery potential will depend critically on the Tracker alignment. As an illustration, in Fig. 3 a hypothetical narrow resonance $X \rightarrow Z Z \rightarrow 4 \mu$ resolution and discovery significance are shown for a scenario of $5 / \mathrm{fb}$ of data with the SM Higgs cross-section and branching fraction parameters.
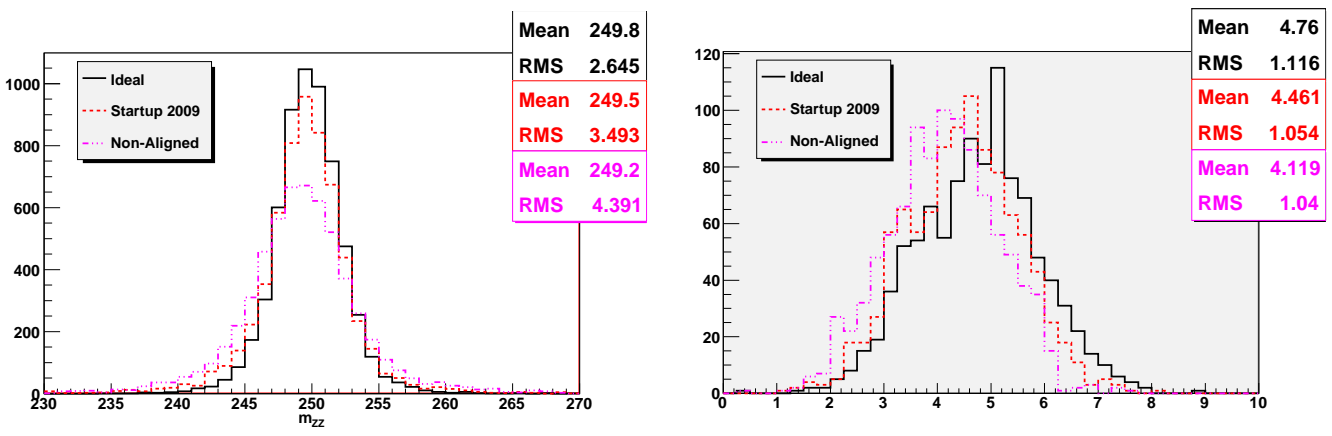

Figure 3: Reconstructed invariant mass (units $\mathrm{GeV} / \mathrm{c}^{2}$ ) of a hypothetical narrow resonance $X \rightarrow Z Z \rightarrow 4 \mu$ (left) and significances of the observed signal (right) with an analysis of a MC sample equivalent to 5/fb of data. Three geometry scenarios are shown: ideal, startup misalignment based on 2008 data performance with cosmic ray data, and non-aligned.

The author would like to thank his colleagues on the CMS experiment, and in particular in the tracker alignment group, for collaboration which produced these results; the US NSF and the A. P. Sloan Foundation for support; and the conference organizers for hospitality.

\section{References}

[1] CMS Tracker Collaboration, JINST 4 T07001, 2009.

[2] V. Blobel, Nucl. Instrum. Meth. A 566, 5, 2006.

[3] V. Karimäki, T. Lampén, and F.-P. Schilling, CMS NOTE-2006/018, 2006.

[4] D. N. Brown, A. V. Gritsan, Z. J. Guo, and D. A. Roberts, Nucl. Instrum. Meth. A 603, 467, 2009. 